\title{
Change in cardiovascular risk factors following bariatric surgery for obesity - First Sri Lankan experience
}

Nayananjani Karunasena ${ }^{1}$, Thejana Wijeratne ${ }^{1}$, Uditha Bulugahapitiya ${ }^{1}$, Pubudu Chulasiri², Kaushalya Kasturiaratchi ${ }^{3}$, Priyanthika Abeysinghe ${ }^{1}$, Waruni AbeysiriGunawardana ${ }^{1}$

${ }^{1}$ Colombo South Teaching Hospital, Colombo, Sri Lanka

${ }^{2}$ National Dengue Control Programme, Colombo, Sri Lanka

3University Of Queensland, Brisbane, Queensland, Australia

\section{Introduction}

- Obesity is associated with increased prevalence of cardiovascular(CV) risk factors and mortality and the CV risk factors are known to reduce following bariatric surgery ${ }^{1}$

- Studies have shown a substantial improvement in lipid abnormalities and risk for $C A D$, which persists for at least 5 to 10 years after bariatric surgery ${ }^{2}$

- The aim of this analysis was to determine the effect of bariatric surgery on CV risk factors and $\mathrm{CV}$ risk scores.

\section{Objectives}

- To assess the cardiovascular risk factors among the patients with obesity.

-To assess the change in cardiovasclur risk factors following sleeve gastrectomy among patients with obesity.

\section{Methodology}

- A prospective analytical study of the first 15 consecutive patients who underwent laparoscopic sleeve gastrectomy (LSG) procedure for obesity from 2009 to 2011 in Colombo.

- Patients with $\mathrm{BMI} \geq 40$ without comobidities and $\mathrm{BMI} \geq 35$ with comobidities who have failed medical therapy and/or lifestyle interventions, were offered laparascopic sleeve gastrectomy.

- Body weight,BMI, systolic (SBP) and diastolic (DBP) blood pressures and lipid profile were recorded preoperatively and repeated at 6 months, 9 months and 12 months following surgery.

- CV risk was calculated using Framingham's CV risk score.

\section{Results}

-Among the studied fifteen patients, 14 were females and one was male.

- Mean age was $40.07+/-10.93$

- Pre operatively 9 patients (60\%) had dyslipidaemia and 7 (46.7\%) had diabetes mellitus and 7 (46.7\%) had hypertension (HT).

•Five (33.3\%) were found to have Impaired Glucose Tolerance (IGT).

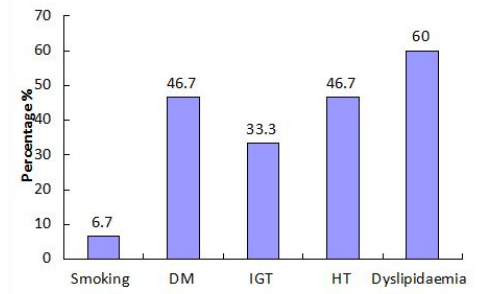

Figure 1 : Cardiovascular risk factors before surgery
- Preoperative mean Body Mass Index (BMI) was 45.1+/-8.09 and showed a $30.6 \%$ reduction $(\mathrm{p}<0.001)$ after one year following surgery.

- SBP showed a $11.2 \%$ reduction $(p<0.01)$ and DBP reduction was $14.5 \%$ $(p<0.01)$

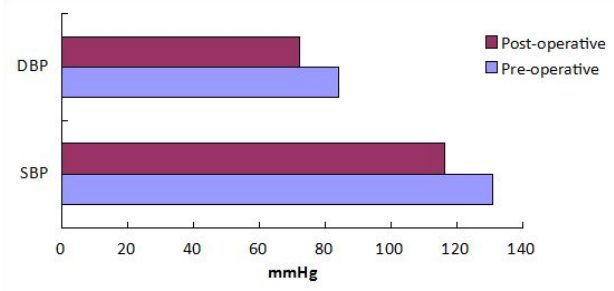

Figure 2: Change in mean systolic and diastolic blood pressures one year following sleeve gastrectomy for obesity

- Mean Triglyceride (TG) level showed a $21.1 \%$ reduction $(p<0.05)$ and HDL cholesterol (HDL-C) level showed a $12.1 \%$ rise after one year from bariatric surgery.

- Total Cholesterol and LDL cholesterol levels did not show a significant reduction.

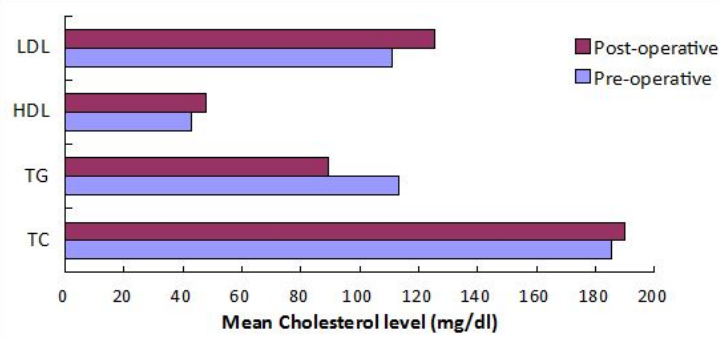

Figure 3: Change in mean cholesterol levels one year following sleeve gastrectomy for obesity

- CV risk score showed a $17.3 \%$ mean reduction at one year follow up, which was not statistically significant.

\section{Conclusions}

- Cardiovascular risk factors, SBP, DBP, TG and HDL-C showed significant improvement 12 months following bariatric surgery.

- $\mathrm{CV}$ risk score showed a statistically non significant reduction after one year following bariatric surgery.

\section{References}

1.Batsis JA, Sarr MG,Maria L. Collazo-Clavell, et al .Cardiovascular risk after bariatric surgery for obesity. American Journal of cardiology 2008; 102(7):930-937.

2.AACE/TOS/ASMBS Bariatric Surgery Guidelines, Endocr Pract. 2008;14(Suppl 1) 